\title{
ULX contribution to stellar feedback: an intermediate-mass black hole candidate and the population of ULXs in the low-metallicity starburst galaxy ESO 338-4 ${ }^{\star}$
}

\author{
L. M. Oskinova ${ }^{1,2}$, A. Bik ${ }^{3}$, J. M. Mas-Hesse ${ }^{4}$, M. Hayes ${ }^{3}$, A. Adamo ${ }^{3}$, G. Östlin ${ }^{3}$, F. Fürst ${ }^{5}$, and H. Otí-Floranes ${ }^{4}$ \\ 1 Institute for physics and astronomy, University of Potsdam, Karl-Liebknecht-Str. 24/25, 14476 Potsdam, Germany \\ e-mail: lida@astro.physik.uni-potsdam.de \\ 2 Department of Astronomy, Kazan Federal University, Kremlevskaya Str 18, Kazan, Russia \\ 3 Department of Astronomy, Stockholm University, Oscar Klein Centre, AlbaNova University Centre, 10691 Stockholm, Sweden \\ 4 Centro de Astrobiología (CSIC-INTA), 28850 Torrejon de Ardoz, Madrid, Spain \\ 5 European Space Astronomy Centre (ESAC), Science Operations Departement, 28692 Villanueva de la Cañada, Madrid, Spain
}

Received 6 March 2019 / Accepted 17 May 2019

\begin{abstract}
Context. X-ray radiation from accreting compact objects is an important part of stellar feedback. The metal-poor galaxy ESO 338-4 has experienced vigorous starburst during the last $<40 \mathrm{Myr}$ and contains some of the most massive super star clusters in the nearby Universe. Given its starburst age and its star-formation rate, ESO 338-4 is one of the most efficient nearby manufactures of neutron stars and black holes, hence providing an excellent laboratory for feedback studies.

Aims. We aim to use X-ray observations with the largest modern X-ray telescopes XMM-Newton and Chandra to unveil the most luminous accreting neutron stars and black holes in ESO 338-4.

Methods. We compared X-ray images and spectra with integral field spectroscopic observations in the optical to constrain the nature of strong X-ray emitters.

Results. X-ray observations uncover three ultraluminous X-ray sources (ULXs) in ESO 338-4. The brightest among them, ESO 338 $\mathrm{X}-1$, has X-ray luminosity in excess of $10^{40} \mathrm{erg} \mathrm{s}^{-1}$. We speculate that ESO 338-4 X-1 is powered by accretion on an intermediate-mass $\left(\gtrsim 300 M_{\odot}\right)$ black hole. We show that X-ray radiation from ULXs and hot superbubbles strongly contributes to He II ionization and general stellar feedback in this template starburst galaxy.
\end{abstract}

Key words. galaxies: dwarf - galaxies: individual: ESO 338-4 - X-rays: binaries - X-rays: ISM

\section{Introduction}

Awareness of the important contribution from high-mass X-ray binaries (HMXBs) to the overall stellar feedback is growing quickly (e.g. Madau et al. 2004; Mirabel et al. 2011; Justham \& Schawinski 2012; Prestwich et al. 2015; Madau \& Fragos 2017). Recently, Sazonov \& Khabibullin (2018) showed that HMXB feedback is likely dominated by ultraluminous X-ray sources (ULXs), which are defined as off-nucleus point sources with X-ray luminosity $L_{\mathrm{X}} \gtrsim 10^{40} \mathrm{erg} \mathrm{s}^{-1}$. In a star forming galaxy in the local Universe, there are about $\sim 0.4-1.2$ ULXs per unit of star formation rate (SFR $M_{\odot} \mathrm{yr}^{-1}$; Mapelli et al. 2010; Sazonov \& Khabibullin 2017). Despite being rare, ULXs in a dwarf galaxy significantly photoionize helium in the interstellar medium (ISM), hence reducing its opacity for the extreme UV and soft X-rays. This leads to a longer mean free path of energetic photons, assisting the ISM heating, and in turn easing the driving of galactic outflows, and facilitating the escape of $\mathrm{LyC}$ and Ly $\alpha$ photons.

It is becoming increasingly clear that the majority of ULXs are powered by super-critical accretion onto neutron stars (NSs) and stellar mass black holes (BHs; see a recent review by Kaaret et al. 2017). The super-critically accreting NS might reach X-ray

* Based on observations obtained with the science missions XMM-Newton (ObsID 0780790201) and Chandra (ObsID 21866). luminosities above $\gtrsim 10^{41} \mathrm{erg} \mathrm{s}^{-1}$ (Israel et al. 2017), but in the ULXs with $L_{\mathrm{X}}>10^{40} \mathrm{erg} \mathrm{s}^{-1}$, accretion onto an intermediatemass black hole (IMBH) is generally suspected (Farrell et al. 2009).

One of the closest $(37.5 \mathrm{Mpc})$ luminous blue compact dwarf galaxies, ESO 338-4, provides an excellent laboratory to better understand and disentangle the various constituencies of stellar feedback. In optical and UV this galaxy shares a number of properties with faint Lyman break galaxies with weak but positive Ly $\alpha$ emission. Bik et al. (2018) presented a comprehensive study of ESO 338-4 and its circumgalactic medium based on integral field spectroscopic observations in optical obtained with the the Multi Unit Spectroscopic Explorer (MUSE) instrument at the Very Large Telescope (VLT); for a complete overview of this galaxy we refer the interested readers to this work and references therein. ESO 338-4 has been undergoing a vigorous starburst for the last $\sim 40 \mathrm{Myr}$ (Östlin et al. 1998, 2003) and contains a large population of young massive star clusters; among them are clusters displaying strong Wolf-Rayet (WR) spectral features. The most remarkable among them is cluster 23 , the most luminous, massive $\left(\sim 10^{7} M_{\odot}\right)$, and young (<10 Myr) super-star cluster (SSC) detected in the nearby galaxies (Östlin et al. 2007). Bik et al. (2018) observed an extended ionized halo around ESO 3384 , SSCs immersed in a large superbubble filled by shocked gas, and a galactic scale wind. Indirect evidence suggests that 


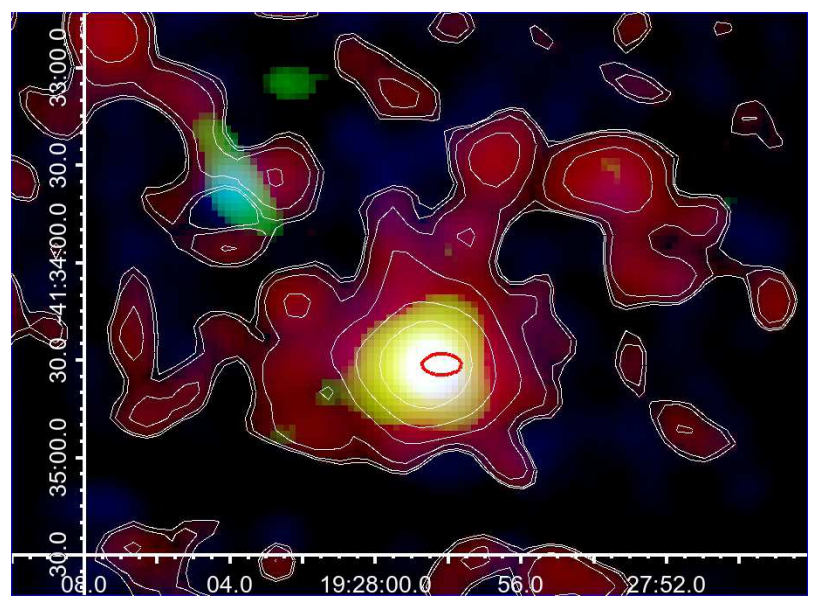

Fig. 1. Combined smoothed XMM-Newton EPIC PN image of ESO 3384 in soft $(0.2-1.0 \mathrm{keV}$, red), medium $(1.0-4.5 \mathrm{keV}$, green) and hard (4.5-12.0 keV, blue) energy bands with the logarithmic-scale contours (white), tracing X-ray emission in the soft band. The image size is $4^{\prime} \times 3^{\prime}$ and north is up and east to the left. The red ellipse $\left(16^{\prime \prime} \times 10^{\prime \prime}\right)$ approximately marks the position and UV extent of the ESO 338-4 galaxy.

ESO 338-4 leaks ionizing LyC radiation (Leitet et al. 2013). The total mass of ESO 338-4 $\left(4 \times 10^{9} M_{\odot}\right)$, its current star formation rate, $3.2 M_{\odot} \mathrm{yr}^{-1}$ (Östlin et al. 2001), and the age of its massive clusters make it one of the most active nearby producers of NSs and BHs.

Here we report XMM-Newton and Chandra X-ray observations of ESO 338-4. In Sect. 2 we present the X-ray observations and X-ray source identifications, while the discussion on the potential IMBH candidate and the contribution of ULXs to stellar feedback in this galaxy is presented in Sect. 3. A summary is provided in Sect. 4.

\section{X-ray observations and source identification}

\subsection{XMM-Newton}

XMM-Newton observed ESO 338-4 on 2016-04-10 (ObsID 0780790201; PI H. Otí-Floranes). We reprocessed these data using the XMM-Newton Science Analysis System (SAS) ${ }^{1}$. The standard analysis steps were performed to obtain the Xray images, spectra, and light curves. After rejecting highbackground time intervals, the useful exposure time was $15 \mathrm{ks}$ for the EPIC PN and $23 \mathrm{ks}$ for the EPIC MOS cameras.

An X-ray image of the sky around ESO 338-4 is shown in Fig. 1. A bright X-ray source is clearly detected at the location of ESO 338-4. This source, 3XMM J192758.7-413430, can be found in the "XMM-Newton Serendipitous Source Catalogue 3 XMM-DR8" at RA $(2000)=19^{\mathrm{h}} 27^{\mathrm{m}} 58.575$, Dec $(2000)=$ $-41^{\circ} 34^{\prime} 30^{\prime \prime} .8$ with an error in position $1^{\prime \prime} .5$. The observed source flux is $(3.2 \pm 0.1) \times 10^{-13} \mathrm{erg} \mathrm{cm}^{-2} \mathrm{~s}^{-1}$. No foreground or background objects coinciding with the X-ray source are seen in the VLT MUSE and Hubble Space Telescope (HST) images (Bik et al. 2018). We conclude that 3XMM J192758.7413430 resides in the ESO 338-4 galaxy. In this case its absorption-corrected X-ray luminosity in $0.2-12.0 \mathrm{keV}$ band is $8 \times 10^{40} \mathrm{erg} \mathrm{s}^{-1}$.

\footnotetext{
1 wWW. cosmos. esa.int/web/xmm-newton/what-is-sas
}

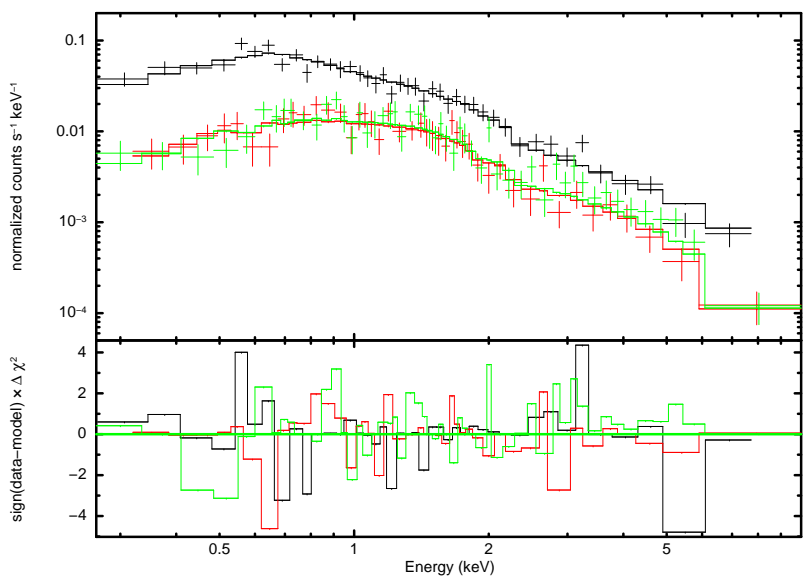

Fig. 2. XMM-Newton EPIC PN, MOS1, and MOS2 spectra of 3XMM J192758.7-413430 (black, red, and green curves, respectively) with error bars corresponding to $3 \sigma$ and the best-fit combined thermal plasma (apec) and power-law model (solid lines). The model parameters are shown in Table 1. Lower panel: residuals between the data and the best-fit model.

Figure 1 shows indications of extended soft X-ray emission surrounding ESO 338-4, as indeed might be expected given the presence of a shocked superbubble and galactic-scale outflows in ESO 338-4 (Bik et al. 2018).

Indirect support for the presence of hot thermal plasma in ESO 338-4 comes from the analysis of low-resolution XMMNewton EPIC spectra (Fig. 2). The spectra were analysed using standard X-ray spectral fitting software xspec (Arnaud 1996). The fitting statistics improve when in addition to a single powerlaw model, a $\sim 2 \mathrm{MK}$ thermal plasma component is included in a spectral mode (reduced $\chi^{2}=0.886$ compared to reduced $\chi^{2}=0.977$ for a simple power-law model). The $F$-test indicates $(F$-value $\approx 7$ and probability 0.001$)$ that including an additional thermal plasma model component is justified. Using a blackbody spectral component instead does not improve fitting statistics compared to a single power-law model (reduced $\chi^{2}=0.969$, $F$-value $\approx 0.6$ and probability 0.5 ). We speculate that soft optically thin thermal plasma X-ray emission is associated with the superbubble and galactic wind.

The power-law component has a spectral index $\Gamma \approx 1.9$ which is similar to the brightest ULXs and is well explained by accretion onto a BH (Sutton et al. 2012).

Interestingly, spectral fits of similar quality could also be achieved with a purely thermal two-temperature model. In this case, a second temperature component with $k T=5.3 \pm 0.6 \mathrm{keV}$ is recovered, which could indicate the presence of a hot accretion disk. To compare with various accretion-disk models we tried the diskpbb model (Kubota et al. 2005) combined with the apec model. A good-quality fit (reduced $\chi^{2} \approx 0.9$ ) is achieved with the temperature at inner disk radius, $k T_{\text {in }}=3.3 \pm 1.5 \mathrm{keV}$ and the exponent of the radial dependence of the disk temperature $p=0.53 \pm 0.02$ (i.e. similar to other ULX spectra; see Pintore et al. 2018).

The foreground Galactic absorption to the galaxy is $N_{\mathrm{H}} \approx$ $5 \times 10^{20} \mathrm{~cm}^{2}$ (Hayes et al. 2005), that is, significantly lower than derived from the X-ray spectra fitting. Bik et al. (2018) determined the extinction map of the the galaxy and found that within the galaxy $E(B-V)$ varies between $\approx 0.05$ and $0.18 \mathrm{mag}$. The largest local absorption in ESO 338-4 could in principle explain the relatively high neutral hydrogen column density derived from the X-ray spectral analysis. 
Table 1. Parameters of the best-fit spectral models to the observed XMM-Newton EPIC spectra of 3XMM J192758.7-413430.

\begin{tabular}{|c|c|}
\hline Model parameter & Best-fit value \\
\hline \multicolumn{2}{|c|}{ tbabs (powerlaw+apec) } \\
\hline$N_{\mathrm{H}}\left(10^{21} \mathrm{~cm}^{-2}\right)$ & $1.5 \pm 0.3$ \\
\hline$k T(\mathrm{keV})$ & $0.22 \pm 0.02$ \\
\hline$E M\left(10^{63} \mathrm{~cm}^{-3}\right)$ & $2 \pm 1$ \\
\hline$\Gamma$ & $1.85 \pm 0.06$ \\
\hline$K$ (photon $\mathrm{keV}^{-1} \mathrm{~cm}^{-2} \mathrm{~s}^{-1}$ at $\left.1 \mathrm{keV}\right)$ & $(5.5 \pm 0.4) \times 10^{-5}$ \\
\hline reduced $\chi^{2}$ for 134 d.o.f. & 0.88 \\
\hline Unabsorbed $F_{\mathrm{X}}^{\text {thermal }}\left(\mathrm{erg} \mathrm{cm}^{-2} \mathrm{~s}^{-1}\right)$ & $7 \times 10^{-14}$ \\
\hline Unabsorbed $F_{\mathrm{X}}^{\text {powerlaw }}\left(\mathrm{erg} \mathrm{cm}^{-2} \mathrm{~s}^{-1}\right)$ & $4 \times 10^{-13}$ \\
\hline \multicolumn{2}{|c|}{ tbabs $\left(N_{\mathrm{H}, 1}\right) *$ powerlaw + tbabs $\left(N_{\mathrm{H}, 2}\right) *$ apec } \\
\hline$N_{\mathrm{H}, 1}\left(10^{21} \mathrm{~cm}^{-2}\right)$ & $0.6 \pm 0.3$ \\
\hline$k T(\mathrm{keV})$ & $0.24 \pm 0.02$ \\
\hline$E M\left(10^{63} \mathrm{~cm}^{-3}\right)$ & $2 \pm 1$ \\
\hline$N_{\mathrm{H}, 2}\left(10^{21} \mathrm{~cm}^{-2}\right)$ & $2.7 \pm 1$ \\
\hline$\Gamma$ & $1.96 \pm 0.1$ \\
\hline$K$ (photon $\mathrm{keV}^{-1} \mathrm{~cm}^{-2} \mathrm{~s}^{-1}$ at $\left.1 \mathrm{keV}\right)$ & $(6.3 \pm 0.7) \times 10^{-5}$ \\
\hline Reduced $\chi^{2}$ for 133 d.o.f. & 0.87 \\
\hline Unabsorbed $F_{\mathrm{X}}^{\text {thermal }}\left(\mathrm{erg} \mathrm{cm}^{-2} \mathrm{~s}^{-1}\right)$ & $7 \times 10^{-14}$ \\
\hline Unabsorbed $F_{\mathrm{X}}^{\text {powerlaw }}\left(\mathrm{erg} \mathrm{cm}^{-2} \mathrm{~s}^{-1}\right)$ & $4 \times 10^{-13}$ \\
\hline Model $F_{\mathrm{X}}\left(\mathrm{erg} \mathrm{cm}^{-2} \mathrm{~s}^{-1}\right)$ & $3 \times 10^{-13}$ \\
\hline Luminosity $L_{\mathrm{X}}\left(\mathrm{erg} \mathrm{s}^{-1}\right)$ & $8 \times 10^{40}$ \\
\hline
\end{tabular}

Notes. Chandra observations (see Sect. 2.2) show that the dominant contribution to the 3XMM J192758.7-413430 X-ray light is provided by the IMBH candidate ESO 338-4 X-1. The fitted models are the thermal plasma apec model for the metallicity $0.2 Z_{\odot}$ combined with a power law with the photon index of power law $\Gamma$, and corrected for ISM absorption tbabs. Fluxes and luminosity are in the $0.2-12.0 \mathrm{keV}$ band, and fluxes are listed for the individual model components as well as for the full model.

However, the high column density of absorbers could also be intrinsic to the ULX itself. To explore this possibility we tested a combined power law plus optically thin thermal plasma model, where each spectral component has its own absorbing column. The fitting results are shown in Table 1 . Indeed, in this case the thermal plasma could be well described as suffering the foreground absorption only, while the power-law component is significantly more strongly absorbed.

The standard timing analysis procedures were employed to search for a period between $0.2 \mathrm{~s}$ and $100 \mathrm{~s}$ in the barycentre and background-corrected X-ray light curves of 3XMM J192758.7413430. No period with sufficiently high statistical significance was uncovered.

\subsection{Chandra}

The angular resolution of the XMM-Newton telescope $\left(\sim 11^{\prime \prime}\right)$ is surpassed by that of the Chandra X-ray telescope $\left(00^{\prime \prime} .5\right)$. Chandra observed ESO 338-4 for 3 ks on 19 Nov 2018 (ObsID 21866, DDT observations). After standard data-reduction steps, the source detection was performed using the wavdetect tool, which accounts for the the point-spread function (PSF). The tool is a part of the Chandra Interactive Analysis of Observations data analysis package $\mathrm{CIAO}^{2}$.

\footnotetext{
2 cxc.harvard.edu/ciao
}

Among the ten sources detected in the whole ACIS-S field of view, three are located within the optical and UV extent of the ESO 338-4 galaxy (Fig. 3). We denote them ESO 338-4 $\mathrm{X}-1, \mathrm{X}-2$, and $\mathrm{X}-3$ in order of decreasing X-ray luminosity as observed on 19 Nov 2018 (Table 2). All three sources are in the ULX luminosity range. The X-ray luminosity of the brightest source, ESO 338-4 X-1, is $L_{\mathrm{X}}(0.5-7 \mathrm{keV}) \approx 4 \times 10^{40} \mathrm{erg} \mathrm{s}^{1}$. Assuming standard accretion, this corresponds to the Eddington luminosity of a $300 M_{\odot} \mathrm{BH}$.

Fewer than approximately 35 counts were detected from ESO 338-4 X-1 (and even less from the other two sources). This is not sufficient for measuring X-ray spectra. However, the hardness ratio is consistent with the spectrum measured by $X M M$ Newton.

No significant time variability was noticed within the $50 \mathrm{~min}$ duration of the Chandra observations. Interestingly, the PSF fitting shows that the "size" of ESO 338-4 X-1 exceeds the size of the PSF by a factor of about two. This might be an indication that the source is extended, for example immersed in a diffuse $\mathrm{X}$-ray-emitting nebula as is sometimes observed around other ULXs (Soria et al. 2010; Webb et al. 2017).

Keeping in mind the huge difference in the sensitivities and angular resolution between XMM-Newton and Chandra exposures, it appears that within the errors the source fluxes did not change between 2016 and 2018 epochs.

\subsection{Potential optical counterparts of $X$-ray sources}

Figure 3 shows an overlay of the optical (HST) images (from Östlin et al. 2009) tracing both the gas and the stars as well as the X-ray (Chandra) contours of ESO 338-4. The 99\% limit on the positional accuracy of Chandra is $1 " .4$. The optical HST images are astrometically aligned to the MUSE datacube using isolated star clusters (see Bik et al. 2018). The accuracy of the MUSE astrometry (calibrated to the USNO UCAC4 catalogue, Zacharias et al. 2013) is estimated to be around 0'.5 or better. Unfortunately, the Chandra exposure was too short to detect any optical foreground stars in X-rays seen by the HST or MUSE - this would have allowed better astrometrical alignment of X-ray, optical and UV images. At the moment, it is difficult to claim a confident identification of UV and optical counterparts of X-ray sources. However, complementing the HST images with the MUSE integral field spectroscopy in the optical is very useful to obtain initial clues as to the nature of the ULXs in ESO 338-4.

The HST data have a completeness limit of about $50 \%$ for a $5 \times 10^{3} M_{\odot}$ cluster at $50 \mathrm{Myr}$ and a $10^{4} M_{\odot}$ cluster at $100 \mathrm{Myr}$ (Östlin et al. 2003), and therefore massive clusters associated with the detected ULXs should be seen in the HST images. The distance between the enigmatic most massive cluster 23 and the X-ray sources X-1 and X-2 is $2^{\prime \prime}$ and $1^{\prime \prime}$, respectively. This is comparable to the positional error of the X-ray sources. Hence we cannot exclude the association between cluster 23 and ESO 338-4 X-1 or X-2.

Nearest to the centroid of the X-ray position of $X-1$ is the cluster 46 (see the nomenclature in Bik et al. 2018) located at $19^{\mathrm{h}} 27^{\mathrm{m}} 58 \mathrm{~s} 56,-41^{\circ} 34^{\prime} 32^{\prime \prime} \cdot 25$. The separation between ESO 338 $4 \mathrm{X}-1$ and cluster 46 is 0 "!31 ( $\sim 55 \mathrm{pc})$ which is smaller than the Chandra angular resolution. The age of the cluster (10 Myr) and its mass $\left(6 \times 10^{4} M_{\odot}\right)$ make it a plausible host of a massive donor star capable of supplying a sufficiently high accretion rate to maintain the nearly Eddington X-ray luminosity of a $\gtrsim 300 M_{\odot} \mathrm{BH}$.

Interestingly, as can be seen in Fig. 3, there is a pronounced arc-like structure to the south of the X-ray-emitting region 


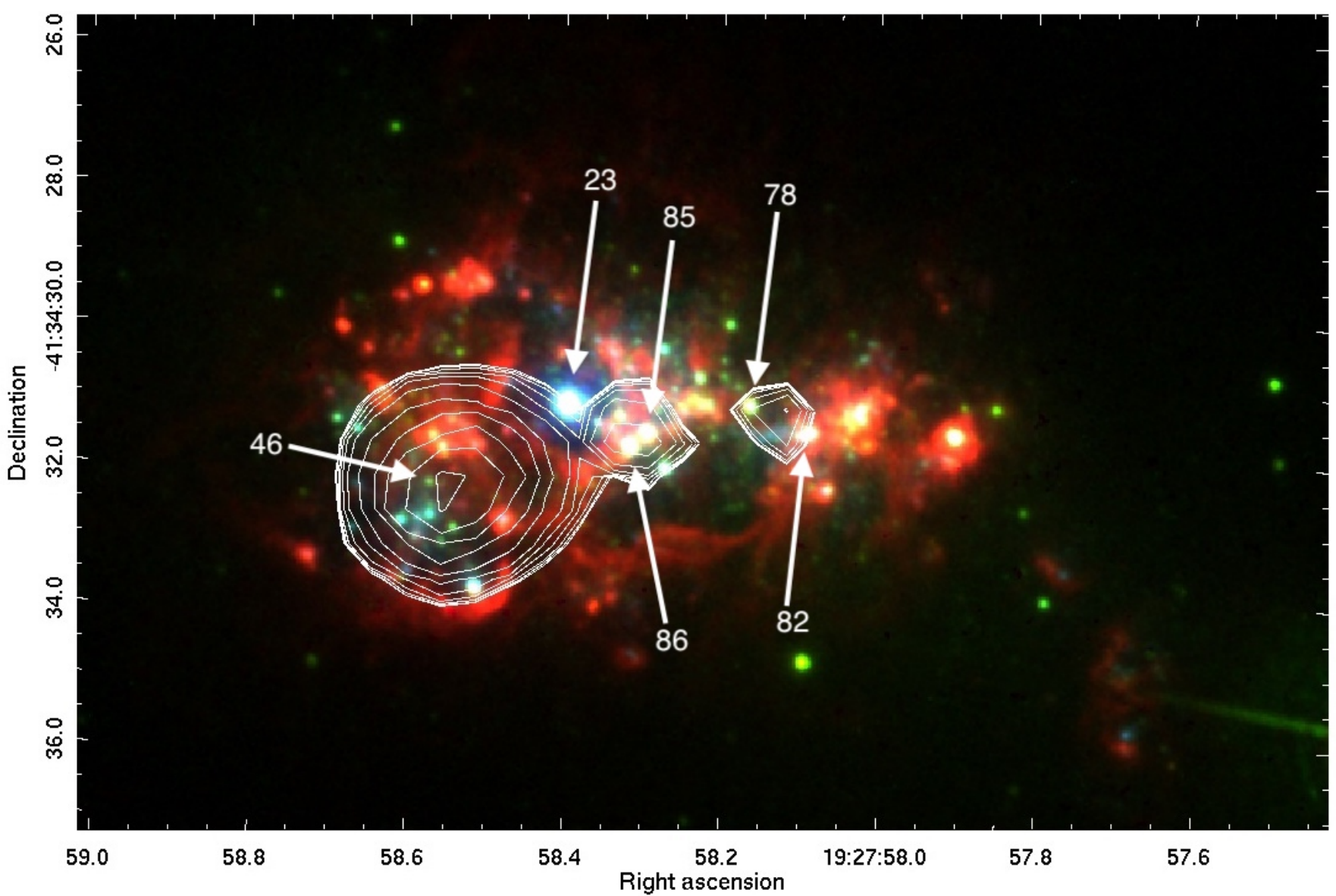

Fig. 3. HST colour composite image of ESO 338-4. The blue shows the image in F140LP UV filter, highlighting the youngest star clusters, green is the F550M filter, and red is the FR656N images centred on the $\mathrm{H} \alpha$ emission line, highlighting the distribution of the ionized gas. Overplotted in white are logarithmically spaced contours tracing the smoothed Chandra X-ray image. Three detected X-ray sources, X-1, X-2, and X-3, are aligned from left to right. The numbered arrows indicate the positions of star clusters that might be associated with the X-ray sources (see discussion in Sect. 2.3 and Table 2.)

Table 2. Parameters of X-ray sources detected by Chandra in the ESO 338-4 galaxy.

\begin{tabular}{ccccccc}
\hline \hline ESO 338-4 & RA & Dec & $\begin{array}{c}\text { Rate }(90 \% \text { confidence) } \\
\left(10^{-3} \mathrm{~s}^{-1}\right)\end{array}$ & $\begin{array}{c}L_{\mathrm{X}} \\
\left(\mathrm{erg} \mathrm{s}^{-1}\right)\end{array}$ & $\begin{array}{c}\text { Nearest cluster } \\
\text { (Bik et al. 2018) }\end{array}$ \\
\hline X-1 & 291.99390 & -41.57566 & $13.10(9.9-17.1)$ & $\sim 4 \times 10^{40}$ & 46 & $0^{\prime \prime} .31$ \\
X-2 & 291.99293 & -41.57542 & $2.21(1-4)$ & $\sim 1 \times 10^{40}$ & $85 / 86$ & $0^{\prime \prime} .19 / 0^{\prime \prime} .21$ \\
X-3 & 291.99219 & -41.57538 & $1.43(0.5-3)$ & $\sim 3 \times 10^{39}$ & $78 / 82$ & $0^{\prime \prime} .4 / 0^{\prime \prime} .5$ \\
\hline
\end{tabular}

Notes. Chandra $99 \%$ limit on positional accuracy is 1".4. Count rates, fluxes (observed), and luminosities (corrected for the ISM absorption) are in $0.5-7.0 \mathrm{keV}$ band. Based on the output from the wavedetect, fluximage, and srcflux tasks which are the part of CIAO package.

containing ESO 338-4 X-1. Future observations should show whether this is an unrelated ISM structure, an outer wall of a superbubble, or a bow shock produced by a jet powered by ESO 338-4 X-1 for example.

Several massive young clusters are found within the positional uncertainty of ESO 338-4 X-2. The closest coincidence is with two massive young clusters: cluster $86\left(19^{\mathrm{h}} 27^{\mathrm{m}} 58^{\mathrm{s}} 308\right.$, $\left.-41^{\circ} 34^{\prime} 31^{\prime \prime} .72\right)$ and cluster $85\left(19^{\mathrm{h}} 27^{\mathrm{m}} 58.286,-41^{\circ} 34^{\prime} 31^{\prime \prime} .53\right)$. Both these clusters are $\sim 6 \mathrm{Myr}$ old and have mass of $\sim 4 \times 10^{5} \mathrm{M}_{\odot}$. MUSE optical spectroscopy of $85 / 86$ clusters shows a hint of a broad He II line, suggestive of WR stars, which would be strongly expected given the cluster ages and masses.

Source ESO 338-4 X-3 also has some young massive clusters within its $1^{\prime \prime} .5$ error circle: clusters $78\left(19^{\mathrm{h}} 27^{\mathrm{m}} 58.156\right.$, $\left.-41^{\circ} 34^{\prime} 31^{\prime \prime} 12,10 \mathrm{Myr}, 5 \times 10^{5} M_{\odot}\right)$ and $82\left(19^{\mathrm{h}} 27^{\mathrm{m}} 58.085\right.$, $\left.-41^{\circ} 34^{\prime} 31^{\prime \prime} 49,4 \mathrm{Myr}, 10^{5} M_{\odot}\right)$. It is tempting to suggest that ESO 338-4 X-2 and ESO 338-4 X-3 might be WR+BH binaries.

\section{Discussion}

\subsection{ESO 338-4 X-1 - an IMBH candidate}

The X-ray luminosity of ESO 338-4X-1 is in the upper range of the ULXs. In a study of 63 ULXs only two were found to be more luminous than $10^{40} \mathrm{erg} \mathrm{s}^{-1}$ (Wolter et al. 2018). Earnshaw et al. (2019) presented a catalogue of non-nuclear $\mathrm{X}$-ray sources in nearby galaxies. Among 384 ULXs in this catalogue, only approximately ten sources in the $40 \mathrm{Mpc}$ 
volume have X-ray luminosities in excess of $4 \times 10^{40} \mathrm{erg} \mathrm{s}^{-1}$. Zolotukhin et al. (2016) presented a search for hyperluminous $\mathrm{X}$-ray sources in the XMM-Newton source catalogue and found about 15 candidates. These latter authors emphasised that only joint optical and X-ray imaging and spectroscopy can confirm their nature.

The X-ray spectrum of ESO 338-4 X-1, a power law with $\Gamma \approx 1.9$, appears to be consistent with other IMBH candidates. Furthermore, ESO 338-4 X-1 is located in a metal-poor galaxy undergoing a vigorous starburst over the last $\sim 40 \mathrm{Myr}$, with a large number of SSCs with ages of $\sim 10 \mathrm{Myr}$. At these ages the most massive stars are already collapsed, while there are still a large number of massive donors at the WR and red supergiant (RSG) evolutionary stages when their mass loss is most prodigious (e.g. Portegies Zwart \& Verbunt 1996; Oskinova 2005).

A causality link between young dense massive star clusters and progenitors of IMBHs has long since been predicted (Begelman \& Rees 1978). IMBHs are expected to be associated with massive star clusters older than $5 \mathrm{Myr}$ (Portegies Zwart \& McMillan 2002). Indeed, detailed simulations show that IMBHs could form at the centres of young, compact stellar clusters (Gürkan et al. 2006). The cluster core collapses relatively quickly ( $\lesssim 5 \mathrm{Myr})$. A runaway sequence of stellar mergers can lead to the formation of a very massive $\operatorname{star}\left(M_{*} \gtrsim 400 M_{\odot}\right)$ (Freitag et al. 2006). Although the final fate of the resulting object is difficult to predict because of uncertainties in stellar evolution, among different potential outcomes is its direct collapse to an IMBH. Recently, Bellovary et al. (2019) performed high-resolution cosmological simulations focusing on dwarf galaxies at zero-redshft. These latter authors found that larger dwarf galaxies are more likely to host massive black holes than those of lower mass. Interestingly, in their simulations, about half of the IMBHs in dwarfs are located within a few kiloparsecs of the galaxy centre. Low-metallicity (low-Z) conditions (but higher than the primordial) are especially favourable for the formation of IMBHs (Mapelli et al. 2010).

So far, IMBHs have not been ambiguously identified (Mezcua 2017). Close to the ESO 243-49 galaxy is the strong IMBH candidate, HLX-1, with a BH of $>10000 M_{\odot}$. The galaxy has modest ongoing star formation in the bulge of only $\sim 0.01 M_{\odot} \mathrm{yr}^{-1}$, and the observations rule out any young SSC close to HLX-1. This unique object might be a nucleus of a satellite dwarf galaxy (Soria et al. 2017).

The discovery of NS accretors in ULXs strongly restricted their diagnostic potential for the IMBHs searches but did not rule out the existence of these latter objects. Nothing we know so far precludes ESO 338-4 X-1 from being a supercritical accreting NS or a $\mathrm{BH}$ of a few tens of solar mass with a favorite beaming orientation. However, given that large theoretical and modeling studies predict that young star clusters with properties well sampled by those in ESO 338-4 provide conditions needed for IMBH formation, we must consider the possibility that ESO 3384 X-1 hosts an IMBH. Furthermore, future observations showing that all ULXs in ESO 338-4 are conventional sources would strongly question our current understanding of massive young cluster evolution and dynamics at low- $Z$.

Observations dictate that ULXs are more common in lowmetallicity galaxies (Prestwich et al. 2013), as indeed predicted theoretically for the ULXs powered by accretion onto black holes (Mapelli et al. 2010). We suggest that the numbers of ULXs containing a NS should have much weaker dependence on the host galaxy metallicity compared to the numbers of ULXs powered by the accretion onto BHs, as also seen in the simulations of the stellar population evolution
(Wiktorowicz et al. 2019). For the BHs in the upper mass range among those detected by the gravitational wave observatories, the ULX-range luminosities could be achieved at certain binary evolutionary stages without the need to invoke supercritical accretion, especially when the donor is a WR-type star (Marchant et al. 2017; Hainich et al. 2018). Given the high star formation rate in the nuclear region of ESO 338-4, a large number of stellar BHs are expected to have formed in the past. The detection of merging episodes of $30-40 M_{\odot}$ BHs by their gravitational wave flashes shows that there are mechanisms by which quite massive BHs could form in massive star-forming regions. Therefore, ULXs powered by accretion onto $20-60 M_{\odot}$ BHs could also be present in ESO 338-4. An alternative scenario would be the formation of an IMBH during a previous star-formation episode in this galaxy. This IMBH might have acquired a donor star only recently.

The apparent location of ESO 338-4 X-1 slightly offset from the most massive clusters in ESO 338-4 is similar to other bright ULXs. For example, Poutanen et al. (2013) showed that ULXs in the Antennae galaxies are not located in the star clusters, and suggested that these ULXs are massive X-ray binaries that have been ejected from clusters by encounters involving several bodies. Egorov et al. (2017) found kinematic evidence of the escape of ULX HoII X-1 from a star cluster. In general, as reviewed by Kaaret et al. (2017), in spiral and moderately starforming dwarf galaxies the ULXs are associated with loose clusters with masses of a few times $10^{3} M_{\odot}$ and ages of $\gtrsim 10 \mathrm{Myr}$, or are located in non-star-forming regions. In large starburst galaxies, bright ULXs are sometimes found near SSCs, for example the IMBH candidate M 82 X-1 with an estimated mass of $M_{\mathrm{BH}} \sim 1000 M_{\odot}$ (Kaaret et al. 2001; Jang et al. 2018). van den Heuvel \& Portegies Zwart (2013) proposed a scenario where accreting IMBHs (with $M_{\mathrm{BH}}=10^{2-3} M_{\odot}$ ) could be companions in massive runaway binaries formed in the dense cores of collapsed star clusters. Moreover, besides a stellar formation channel, other possible formation channels might be expected in a tidally interacting galaxy, such as ESO 338-4. The improved astrometrical alignment of the HST and deeper Chandra images should help to clarify the association between ULXs and star clusters in ESO 338-4.

Overall, given the present day observations and according to the criteria discussed in Sutton et al. (2012), ESO 338-4 X-1 appears to be an eligible accreting IMBH candidate.

\subsection{Contribution of ULXs to stellar feedback in ESO338-4}

Using the advantages of integral field spectroscopy, Bik et al. (2018) derived ionization maps of ESO 338-4 and its circumgalactic matter. They found that the central area of ESO 338-4 is highly ionized, showing very high ratios in $[\mathrm{O}$ III $] /[\mathrm{S}$ II $]$ and $[\mathrm{O}$ III $] / \mathrm{H} \alpha$ (see their Fig. 7 and corresponding discussion in their Sect. 4.4). The ionization potential of $\mathrm{O}^{+}$is $I_{\mathrm{p}}>35.2 \mathrm{eV}$. The spatial extent of the area with very high $\left[\mathrm{O}_{\mathrm{III}}\right] /[\mathrm{S}$ II] strongly overlaps with the area filled by the nebular He II emission, indicating very high ionization. The total He II emission, including both broad and narrow emission line components, is $\approx 1.1 \times$ $10^{-14} \mathrm{erg} \mathrm{s}^{-1} \mathrm{~cm}^{-2}$, which corresponds to $L_{\mathrm{HeII}} \approx 2 \times 10^{39} \mathrm{erg} \mathrm{s}^{-1}$. Interestingly, the $\left[\mathrm{O}_{\mathrm{III}}\right] /\left[\mathrm{S}_{\mathrm{II}}\right]$ ratio is higher for lower optical depths of LyC photons (Pellegrini et al. 2012). Our X-ray observations showed that the area of high ionization in ESO 338-4 is affected by strong X-ray ionizing field from the ULXs and likely the diffuse hot plasma. Existing X-ray observations detect only the brightest X-ray sources, while missing a large population of the fainter ones; for example, according to the cluster 
wind models, the cluster 23 alone should drive a hot cluster wind with $L_{\mathrm{X}} \gtrsim 10^{37} \mathrm{erg} \mathrm{s}^{-1}$ (Chevalier \& Clegg 1985; Oskinova 2005), which is below the sensitivity of current Chandra observations.

To estimate how many He II ionizing photons are produced by X-ray sources we need to know their flux in the $0.0544-0.25 \mathrm{keV}$ energy band. For this purpose we used the diskpbb+apec spectral model (see Sect. 2.1) which better accounts for the flattening of the ULX spectra at low energies. The unabsorbed flux is $F_{0.0544-0.25 \mathrm{keV}} \approx 1 \times 10^{-13} \mathrm{erg} \mathrm{s}^{-1} \mathrm{~cm}^{-2}$, corresponding to $L_{0.0544-0.25 \mathrm{keV}} \approx 2 \times 10^{40} \mathrm{erg} \mathrm{s}^{-1}$. Integrating the unabsorbed model spectrum over the $0.0544-0.25 \mathrm{keV}$ energy interval yields the number of He II ionizing photons $3 \times 10^{50} \mathrm{~s}^{-1}$. Assuming a branching ratio of five to produce a photon in the He II $\lambda 4686 \AA$ line, the predicted luminosity of ESO $338-4$ in the He II line is $\sim 1 \times 10^{39} \mathrm{erg} \mathrm{s}^{-1}$ which is comparable to the observed one.

X-rays from hot thermal plasma (described by the apec spectral component) dominate He II ionization. Neglecting the thermal plasma radiation and accounting only for the ULX spectrum (as described by the diskpbb model) is responsible for only about $5 \%$ of He II luminosity, $\sim 4 \times 10^{37} \mathrm{erg} \mathrm{s}^{-1}$. In our model estimates we neglect local absorption. Although the metal-poor gas in ESO 338-4 is especially transparent for soft $\mathrm{X}$-rays, our estimate of $\mathrm{He}$ II ionizing photons is likely an upper limit.

Hot $\mathrm{O}$ and WR stars, which are abundant in this galaxy, further contribute to $\mathrm{He}$ II ionization. For example, a hot $\mathrm{O}$ star with $T_{\text {eff }}=56 \mathrm{kK}$ at $Z=0.2 Z_{\odot}$ produces $10^{47} \mathrm{~s}^{-1} \mathrm{He}$ II ionizing photons, while an order of magnitude higher number of ionizing photons is produced by the hottest WN-type stars $\left(T_{\text {eff }}=140 \mathrm{kK}\right.$ at $\left.Z=0.2 Z_{\odot}\right)^{3}$. Given the large number of $\mathrm{O}$ and WR stars in ESO 338-4, and including soft X-rays, there is no need for exotic very massive or stripped stars to explain the ionization of ESO 338-4.

Recently, Schaerer et al. (2019) concluded that both empirical data and theoretical models agree that high-mass $\mathrm{X}$ ray binaries are the main source of nebular He II emission in low-Z star-forming galaxies. At present, our study of ESO 3384 shows that the dominant agent of He II ionization is soft Xrays, likely emitted by shocked gas in superbubbles around star clusters. However, much deeper observations of this galaxy are required in order to make any firm conclusions.

Lebouteiller et al. (2017) suggested that X-ray heating quenches star formation on large scales in galaxies. It seems that in galaxies like ESO 338-4, heating and ionization by X-rays could hinder sequential star formation.

The number of ULXs detected in ESO 338-4 is in a agreement with the expectations from the universal X-ray luminosity function which strongly depends on galactic SFR (Grimm et al. 2003), and is consistent with other SFR indicators. We should note that ESO 338-4 provides an important constraint for the calibrations of the dependence of the X-ray luminosity function on metallicity, which is presently not well known.

\section{Summary}

XMM-Newton and Chandra observations identified three ULXs in ESO 338-4, a galaxy which has been experiencing a strong

The rate of $\mathrm{H}, \mathrm{He}$ II, and $\mathrm{He}$ III ionizing photons could easily be retrieved from the public library of $\mathrm{O}$ and WR model atmospheres PoWR www.astro.physik.uni-potsdam.de/PoWR (Todt et al. 2015; Hainich et al. 2019). episode of massive star formation for the last $40 \mathrm{Myr}$. Given the large number of young, 10 Myr-old SSCs in ESO 338-4 and the large number of theoretical studies that independently predict the formation of IMBHs in SSCs of similar ages and metallicity, we propose that the brightest ULX ESO 338-4 $\mathrm{X}-1$, with a luminosity of $\gtrsim 4 \times 10^{40} \mathrm{erg} \mathrm{s}^{-1}$ is an IMBH candidate. While the Chandra astrometric accuracy is not high enough to firmly associate the ULXs to some of the SSCs previously identified in ESO 338-4, the proximity of the ULXs to SSCs makes it very plausible that the ULXs are powered by the massive star donors. From XMM-Newton observations, we have found some indications for the presence of extended soft X-ray emission surrounding ESO 338-4, which would be compatible with the presence of a shocked superbubble. Finally, the hard ionizing flux produced by ULXs, together with the soft X-ray emission from the superbubbles and the ionization produced by the abundant WR stars in this region, could explain the extended He II emission and the high level of ionization of the central area of ESO 338-4. We conclude, that the contribution of ULXs to stellar feedback in the metal-poor starburst galaxy ESO 338-4 is highly significant.

Acknowledgements. The authors thank the referee for their very useful comments and suggestions which allowed to significantly improve the paper. The authors are grateful for the community response following the arXiv posting of the submitted manuscript - numerous comments and suggestions were incorporated into the manuscript and strongly improved it. The authors are grateful to Chandra observatory's Director for the DDT allocation. LMO acknowledges financial support by the Deutsches Zentrum für Luft und Raumfahrt (DLR) grant FKZ 50 OR 1809, and partial support by the Russian Government Program of Competitive Growth of Kazan Federal University. JMMH is funded by Spanish State Research Agency grants ESP2017-87676-C5-1-R and MDM-2017-0737 (Unidad de Excelencia 'María de Maeztu' CAB). A.A., A.B., M.H., and G.Ö. acknowledge the support of the Swedish Research Council, Vetenskapsrådet and the Swedish National Space Agency (SNSA).

\section{References}

Arnaud, K. A. 1996, in Astronomical Data Analysis Software and Systems V, eds. G. H. Jacoby, \& J. Barnes, ASP Conf. Ser., 101, 17

Begelman, M. C., \& Rees, M. J. 1978, MNRAS, 185, 847

Bellovary, J. M., Cleary, C. E., Munshi, F., et al. 2019, MNRAS, 482, 2913

Bik, A., Östlin, G., Menacho, V., et al. 2018, A\&A, 619, A131

Chevalier, R. A., \& Clegg, A. W. 1985, Nature, 317, 44

Earnshaw, H. P., Roberts, T. P., Middleton, M. J., Walton, D. J., \& Mateos, S. 2019, MNRAS, 483, 5554

Egorov, O. V., Lozinskaya, T. A., \& Moiseev, A. V. 2017, MNRAS, 467, L1

Farrell, S. A., Webb, N. A., Barret, D., Godet, O., \& Rodrigues, J. M. 2009, Nature, 460, 73

Freitag, M., Gürkan, M. A., \& Rasio, F. A. 2006, MNRAS, 368, 141

Grimm, H.-J., Gilfanov, M., \& Sunyaev, R. 2003, MNRAS, 339, 793

Gürkan, M. A., Fregeau, J. M., \& Rasio, F. A. 2006, ApJ, 640, L39

Hainich, R., Oskinova, L. M., Shenar, T., et al. 2018, A\&A, 609, A94

Hainich, R., Ramachandran, V., Shenar, T., et al. 2019, A\&A, 621, A85

Hayes, M., Östlin, G., Mas-Hesse, J. M., et al. 2005, A\&A, 438, 71

Israel, G. L., Belfiore, A., Stella, L., et al. 2017, Science, 355, 817

Jang, I., Gliozzi, M., Satyapal, S., \& Titarchuk, L. 2018, MNRAS, 473, 136

Justham, S., \& Schawinski, K. 2012, MNRAS, 423, 1641

Kaaret, P., Prestwich, A. H., Zezas, A., et al. 2001, MNRAS, 321, L29

Kaaret, P., Feng, H., \& Roberts, T. P. 2017, ARA\&A, 55, 303

Kubota, A., Ebisawa, K., Makishima, K., \& Nakazawa, K. 2005, ApJ, 631, 1062

Lebouteiller, V., Péquignot, D., Cormier, D., et al. 2017, A\&A, 602, A45

Leitet, E., Bergvall, N., Hayes, M., Linné, S., \& Zackrisson, E. 2013, A\&A, 553, A106

Madau, P., \& Fragos, T. 2017, ApJ, 840, 39

Madau, P., Rees, M. J., Volonteri, M., Haardt, F., \& Oh, S. P. 2004, ApJ, 604, 484

Mapelli, M., Ripamonti, E., Zampieri, L., Colpi, M., \& Bressan, A. 2010, MNRAS, 408, 234

Marchant, P., Langer, N., Podsiadlowski, P., et al. 2017, A\&A, 604, A55

Mezcua, M. 2017, Int. J. Mod. Phys. D, 26, 1730021 
Mirabel, I. F., Dijkstra, M., Laurent, P., Loeb, A., \& Pritchard, J. R. 2011, A\&A, 528, A149

Oskinova, L. M. 2005, MNRAS, 361, 679

Östlin, G., Bergvall, N., \& Roennback, J. 1998, A\&A, 335, 85

Östlin, G., Amram, P., Bergvall, N., et al. 2001, A\&A, 374, 800

Östlin, G., Zackrisson, E., Bergvall, N., \& Rönnback, J. 2003, A\&A, 408, 887

Östlin, G., Cumming, R. J., \& Bergvall, N. 2007, A\&A, 461, 471

Östlin, G., Hayes, M., Kunth, D., et al. 2009, AJ, 138, 923

Pellegrini, E. W., Oey, M. S., Winkler, P. F., et al. 2012, ApJ, 755, 40

Pintore, F., Zampieri, L., Mereghetti, S., et al. 2018, MNRAS, 479, 4271

Portegies Zwart, S. F., \& McMillan, S. L. W. 2002, ApJ, 576, 899

Portegies Zwart, S. F., \& Verbunt, F. 1996, A\&A, 309, 179

Poutanen, J., Fabrika, S., Valeev, A. F., Sholukhova, O., \& Greiner, J. 2013, MNRAS, 432, 506

Prestwich, A. H., Tsantaki, M., Zezas, A., et al. 2013, ApJ, 769, 92

Prestwich, A. H., Jackson, F., Kaaret, P., et al. 2015, ApJ, 812, 166
Sazonov, S., \& Khabibullin, I. 2017, MNRAS, 466, 1019 Sazonov, S., \& Khabibullin, I. 2018, MNRAS, 476, 2530 Schaerer, D., Fragos, T., \& Izotov, Y. I. 2019, A\&A, 622, L10 Soria, R., Pakull, M. W., Broderick, J. W., Corbel, S., \& Motch, C. 2010, MNRAS, 409, 541

Soria, R., Musaeva, A., Wu, K., et al. 2017, MNRAS, 469, 886

Sutton, A. D., Roberts, T. P., Walton, D. J., Gladstone, J. C., \& Scott, A. E. 2012, MNRAS, 423, 1154

Todt, H., Sander, A., Hainich, R., et al. 2015, A\&A, 579, A75

van den Heuvel, E. P. J., \& Portegies Zwart, S. F. 2013, ApJ, 779, 114

Webb, N. A., Guérou, A., Ciambur, B., et al. 2017, A\&A, 602, A103

Wiktorowicz, G., Lasota, J.-P., Middleton, M., \& Belczynski, K. 2019, ApJ, 875, 53

Wolter, A., Fruscione, A., \& Mapelli, M. 2018, ApJ, 863, 43

Zacharias, N., Finch, C. T., Girard, T. M., et al. 2013, AJ, 145, 44

Zolotukhin, I., Webb, N. A., Godet, O., Bachetti, M., \& Barret, D. 2016, ApJ, 817,88 